\title{
TMA: Tell Me About It!
}
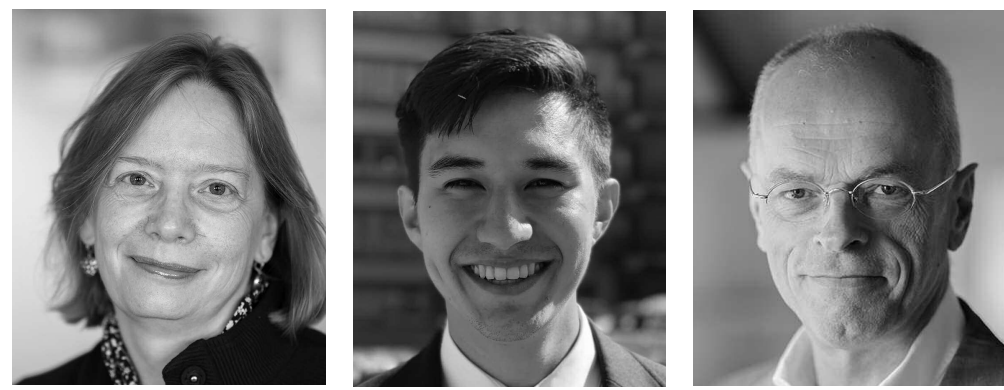

During a gathering of the United States \& Canadian Academy of Pathology in March 2019, where we celebrated the publication of a new edition of a book on renal histology, a discussion about "TMA" arose. Surya V. Seshan of Weill Cornell Medicine, New York, described a case she recently encountered with the words "Tell Me About It!" And Bob Colvin of Massachusetts General Hospital/Harvard Medical School interrupted: "Yes, that's TMA - tell me about it!"

In this issue of The Journal, Li, et al ${ }^{1}$ report on thrombotic microangiopathy (TMA) in lupus nephritis, which is, as the authors state, a relatively uncommon pathological finding in lupus nephritis. Its clinical significance remains to be defined. In their study, TMA was present in $24(3.5 \%)$ of 677 biopsy-proven lupus nephritis cases. Previous studies have also found that TMA is a relatively uncommon finding in lupus nephritis, with a prevalence usually below $10 \%$, although some studies report TMA to be present in up to 25\% of renal biopsies with lupus nephritis ${ }^{2}$. The reported variability of TMA in lupus nephritis may depend on numerous factors including the clinical investigation of patients with systemic lupus erythematosus (SLE), their (genetic) background or geography, but also with the way in which TMA was tested for and evaluated. All histological findings in the study by $\mathrm{Li}$, et al were reevaluated, which led to exclusion of 2 cases in which the histology did not show convincing evidence of renal TMA; by reevaluation no additional cases with TMA were identified. Definitions for TMA vary considerably (in lupus nephritis as well as in other conditions), and careful reevaluation of renal biopsies - not only those with lupus nephritis but also with other diseases - may reveal more or fewer TMA lesions than were initially appreciated.

$\mathrm{Li}$, et al define TMA as endothelial swelling, lumen narrowing, or obliteration and thrombi formation ${ }^{1}$. From this definition it is slightly unclear whether the presence of thrombi is the sine qua non for a biopsy to be considered as TMA-positive. There is a recent tendency to name certain vascular lesions as microangiopathy, in which fibrin thrombi may or may not be present $^{3}$, to encompass the whole spectrum of lesions ranging from changes most likely preceding actual fibrin thrombus formation, to more chronic lesions when the actual fibrin thrombus has been resolved. In fact, this distinction was also made in the publication by Hughson, et $a l^{4}$ to which Li, et al refer as the basis for their definition. In 1992, Hughson and colleagues described 3 patients, 2 with SLE and 1 with primary antiphospholipid syndrome, who developed acute renal insufficiency with thrombocytopenia. The vascular lesions in the renal biopsies of these patients were characterized by thrombi or by cellular and mucoid intimal hyperplasia of small arteries and arterioles. Moreover, noninflammatory lupus microangiopathy was ruled out by the absence of subendothelial immunoglobulin deposits. In that article of more than 25 years ago, Hughson, et $a l^{4}$ express the hope that as more knowledge is gained, the accurate identification of renal vascular lesions in SLE or related diseases could influence renal prognosis and choice of therapy.

Since 1992, numerous studies have appeared that addressed the issue of vascular lesions in SLE in general, and TMA in lupus nephritis in particular. $\mathrm{Li}$, et al argue that reported findings on TMA are variable and inconclusive ${ }^{1}$, with reference to 3 studies $^{5,6,7}$. Of these 3 studies, the one by Barrera-Vargas, $e t a l^{7}$ is very similar to the study by $\mathrm{Li}$, et al, investigating risk factors for TMA in 23 patients with TMA on renal biopsy out of a total group of 235 patients with lupus nephritis and comparing the TMA-positive group with a control group ${ }^{7}$. Lymphopenia and anti-Ro positivity were shown to be independent risk factors for renal TMA and the authors hypothesize that they could have led to endothelial dysfunction and damage. There were no differences in Systemic Lupus Erythematosus Disease Activity Index score, endstage renal disease, or mortality between the 23 patients with renal TMA and 21 control subjects in this study. However, in what appears to be a followup study ${ }^{8}$ led

See TMA in lupus nephritis, page 1478

Personal non-commercial use only. The Journal of Rheumatology Copyright (C) 2019. All rights reserved. 
by some of the authors of the study by Barrera-Vargas, 429 patients with lupus nephritis were reported from the same hospital 1 year later, with TMA present in a similar number of 23. In this study, other groups with renal vascular pathology in lupus nephritis were distinguished from those with TMA; they had arterial sclerosis, noninflammatory necrotizing vasculitis, and true renal vasculitis. Slightly fewer than half the patients had no vascular lesions, and these patients were younger and had better renal function. Five-year renal survival was significantly worse in patients in whom lupus nephritis was accompanied by TMA or noninflammatory necrotizing vasculitis than in those without vascular lesions or other vascular lesions. A similar distinction between various vascular lesions (i.e., vascular lesions with immune complex deposits, atherosclerosis, TMA, noninflammatory necrotizing vasculopathy, and true renal vasculitis) was made by $\mathrm{Wu}$, et $a l^{5}$, showing that patients with TMA had the poorest renal outcome. Another publication from the same center and in the same year took a closer look at the spectrum of TMA in lupus nephritis ${ }^{6}$, demonstrating that there were various causes of TMA in lupus nephritis: thrombotic thrombocytopenic purpura, hemolytic uremic syndrome, antiphospholipid syndrome, malignant hypertension, systemic sclerosis, and so-called isolated renal TMA. In that study by Song, et $a l^{6}$, it was also suggested that complement overactivation through both classical and alternative pathways might play an important role in the pathogenesis of renal TMA in lupus nephritis.

Although fragmentary, recent studies have focused on exactly this topic: can TMA in lupus nephritis be attributed to a variety of causes, and what is the role of the complement system in all or some of these forms of TMA? And most importantly: does more in-depth knowledge about the cause of TMA in lupus nephritis provide us with a rationale for therapeutic management? Recent studies focused in particular on TMA in lupus nephritis in relation to antiphospholipid antibodies (aPL), ADAMTS13 inhibitor profiles, and so-called complement mediated TMA; the results are briefly discussed below.

In a recent study by Sciascia, et $a l^{9}, 97$ patients were included with biopsy-proven lupus nephritis accompanied by TMA; a distinction by histology subdivided the group in 42 patients with acute and 55 patients with chronic TMA. Sixty-one patients had aPL and of those, 37 received anticoagulants, which was associated with a higher rate of complete response. In a multivariate analysis limited to the aPL-positive subgroup, variables significantly associated with complete and partial response were acute rather than chronic TMA and the use of vitamin K antagonists/heparin. For the entire group of 97 patients, chronic features of TMA was one of 3 variables associated with absence of kidney response.

An interesting finding was reported in The Journal by Yue, et $a l^{10}$. They investigated characteristics and outcomes of patients with lupus nephritis based on their ADAMTS13 inhibitor profiles. ADAMTS13 inhibitor and severe ADAMTS13 deficiency were detected in 6 of 19 patients with TMA and active SLE, which suggests that these patients could have a thrombotic thrombocytopenic purpura (TTP)-like condition in combination with SLE.

Park, et al reflected on a subset of lupus patients with clinical and pathological manifestations of atypical hemolytic uremic syndrome (HUS) ${ }^{11}$, based on their finding that complement inhibition was a successful treatment in 10 of 11 patients with a variety of causes that led to complement dysregulation, and in whom infection was the most common cause of what these authors called complement-mediated TMA. Although it is unfortunate that this regular article lacks an accurate description of renal biopsy findings, the study gives impetus to further investigations into the prevalence of generally unsuspected etiologies of TMA in lupus nephritis such as complement dysregulation as well as the consequences in forms of therapeutic options.

Given the recent evidence, it would be interesting to hypothesize on all the numerous possible causes for TMA in lupus nephritis. In fact, the association of TMA with anti-Ro antibodies found by $\mathrm{Li}$, et $a l^{1}$, and previously reported by others, may be a clue to yet another subgroup of patients with TMA and lupus nephritis. At the same time, knowledge about the role of the complement system in the development of TMA is rapidly growing. In renal biopsies with TMA from various causes, we found evidence of complement activation through C4d deposits in $88 \%{ }^{12}$. An updated overview of key pathophysiological processes underpinning complement activation and dysregulation in TMA was recently published by Gavriilaki, et $a l^{13}$, and they also conclude that in more and more entities, such as TMA associated with lupus nephritis, complement dysregulation plays a role.

Not all lupus nephritis biopsies have signs of TMA; vascular lesions may be absent, or there may be vascular lesions other than TMA. The histology of TMA is variable and so are its definitions. It is questionable whether SLE-related TMA truly exists. Recent publications have listed multiple causes and differential diagnoses for $\mathrm{TMA}^{13,14}$, and in their listings, SLE is mentioned as a separate entity. A slightly different viewpoint is taken here - that all known causes for TMA should be considered in a patient with TMA accompanying SLE, and that perhaps "SLE-related TMA" does not exist. Studies focusing on a particular cause of TMA in lupus nephritis tend to refer to patients in whom this cause is absent as having "TMA attributable to SLE." But perhaps all TMA in SLE is related to a specific cause that is only found if it is meticulously sought after. In a vast proportion of lupus nephritis, TMA will occur in the presence of aPL, raising the question whether these patients have both lupus nephritis and antiphospholipid nephropathy ${ }^{15}$ as comorbidities. Following this line of argument, other causes of TMA in lupus nephritis biopsies

Personal non-commercial use only. The Journal of Rheumatology Copyright @ 2019. All rights reserved. 
could be considered as comorbidities as well. Thus, patients could have lupus nephritis and TTP, lupus nephritis and infection, lupus nephritis and atypical HUS, etc. Recent findings suggest that complement activation plays a role in the vast majority of all TMA regardless of its cause. Therapies addressing the complement system may be useful for patients with TMA where this is the case. The findings by $\mathrm{Li}$, et al show that the prognosis of lupus nephritis accompanied by TMA is worse than if TMA is absent ${ }^{1}$. Future studies should focus on causes of TMA in lupus nephritis and the ways in which it should be treated.

INGEBORG M. BAJEMA, $M D, P h D$, Pathologist, Department of Pathology, Leiden University Medical Center;

JAMIE S. CHUA, MD, PhD fellow, Department of Pathology, Leiden University Medical Center;

JAN A. BRUIJN, $\mathrm{MD}, \mathrm{PhD}$,

Pathologist, Department of Pathology, Leiden University Medical Center, Leiden, the Netherlands.

Address correspondence to Dr. I.M. Bajema, Department of Pathology, Leiden University Medical Center, L1-Q, PO Box 9600, 2300 RC Leiden, the Netherlands.E-mail: i.bajema@lumc.nl

\section{REFERENCES}

1. Li C, Yap D, Chan G, Wen YB, Li H, Tang C, et al. Clinical outcomes and clinicopathological correlations in lupus nephritis with kidney biopsy showing thrombotic microangiopathy. J Rheumatol 2019;46:1478-84.

2. Pattanashetti N, Anakutti H, Ramachandran R, Rathi M, Sharma A, Nada R, et al. Effect of thrombotic microangiopathy on clinical outcomes in Indian patients with lupus nephritis. Kidney Int Rep 2017;2:844-9.

3. Goodship TH, Cook HT, Fakhouri F, Fervenza FC, Frémeaux-Bacchi V, Kavanagh D, et al; conference participants. Atypical hemolytic uremic syndrome and $\mathrm{C} 3$ glomerulopathy: conclusions from a "Kidney Disease: Improving Global Outcomes" (KDIGO) Controversies Conference. Kidney Int 2017;91:539-51.

4. Hughson MD, Nadasdy T, McCarty GA, Sholer C, Min KW, Silva F. Renal thrombotic microangiopathy in patients with systemic lupus erythematosus and the antiphospholipid syndrome. Am J Kidney Dis 1992;20:150-8.

5. Wu LH, Yu F, Tan Y, Qu Z, Chen MH, Wang SX, et al. Inclusion of renal vascular lesions in the 2003 ISN/RPS system for classifying lupus nephritis improves renal outcome predictions. Kidney Int 2013;83:715-23.

6. Song D, Wu LH, Wang FM, Yang XW, Zhu D, Chen M, et al. The spectrum of renal thrombotic microangiopathy in lupus nephritis. Arthritis Res Ther 2013;15:R12.

7. Barrera-Vargas A, Rosado-Canto R, Merayo-Chalico J, Arreola-Guerra JM, Mejia-Vilet JM, Correa-Rotter R, et al. Renal thrombotic microangiopathy in proliferative lupus nephritis: risk factors and clinical outcomes: a case-control study. J Clin Rheumatol 2016;22:235-40.

8. Mejía-Vilet JM, Córdova-Sánchez BM, Uribe-Uribe NO, Correa-Rotter R, Morales-Buenrostro LE. Prognostic significance of renal vascular pathology in lupus nephritis. Lupus 2017;26:1042-50.

9. Sciascia S, Yazdany J, Dall'Era M, Fenoglio R, Radin M, Aggarwal $\mathrm{I}$, et al. Anticoagulation in patients with concomitant lupus nephritis and thrombotic microangiopathy: a multicentre cohort study. Ann Rheum Dis 2018 Dec 14 (E-pub ahead of print).

10. Yue C, Su J, Gao R, Wen Y, Li C, Chen G, et al. Characteristics and outcomes of patients with systemic lupus erythematosus-associated thrombotic microangiopathy, and their acquired ADAMTS13 inhibitor profiles. J Rheumatol 2018;45:1549-56.

11. Park MH, Caselman N, Ulmer S, Weitz IC. Complement-mediated thrombotic microangiopathy associated with lupus nephritis. Blood Adv 2018;2:2090-4

12. Chua JS, Baelde HJ, Zandbergen M, Wilhelmus S, van Es LA, de Fijter JW, et al. Complement factor $\mathrm{C} 4 \mathrm{~d}$ is a common denominator in thrombotic microangiopathy. J Am Soc Nephrol 2015; 26:2239-47

13. Gavriilaki E, Anagnostopoulos A, Mastellos DC. Complement in thrombotic microangiopathies: unraveling Ariadne's thread into the labyrinth of complement therapeutics. Front Immunol 2019;10:337.

14. Bayer G, von Tokarski F, Thoreau B, Bauvois A, Barbet C, Cloarec $\mathrm{S}$, et al. Etiology and outcomes of thrombotic microangiopathies. Clin J Am Soc Nephrol 20195;14:557-66.

15. de Azevedo FVA, Maia DG, de Carvalho JF, Rodrigues CEM. Renal involvement in antiphospholipid syndrome. Rheumatol Int. 2018;38:1777-89.

J Rheumatol 2019;46:1425-7; doi:10.3899/jrheum.190447 